\title{
Block Classification and Transmission of Compressed Image with Bit Allocation and Unequal Error Protection
}

\author{
Jigisha N. Patel, Suprav Patnaik, and Saraiya Mansi
}

\begin{abstract}
In image/video transmission system block or macro block classification has frequently been used to classify similar types of properties of block like spectral, statistical, perceptual etc. The bit allocation algorithm distribute total number of bits among a finite set of quantizer to maximize the quality. This paper proposed joint source channel coding (JSCC) algorithm for image transmission with: (i) optimum bit allocation for classified blocks (ii) Unequal Error Protection (UEP) at channel coding stage to minimize the total distortion. The bit allocation applied at two levels. First distribute allocate source rate between three different classes and second each class individual transforms coefficient bit assignment. The simulation results shows that using this algorithm approximately 3-4 dB improvement compare to conventional UEP and further 1.5-2 dB improvement using optimum bit allocation algorithm.
\end{abstract}

Index Terms-DCT, JPEG compression, JSCC, UEP, EMNSD.

\section{INTRODUCTION}

Digital images are compressed because of the limitation of the storage size and available channel bandwidth. The Discrete Cosine Transform (DCT) [1] is the heart of image and video compression standards such as JPEG, MPEG-x, H.26x, HDTV etc. In standard JPEG, input image blocks allow transformation, quantization and entropy coding procedure. So bit allocation is irrespective of significance of block [1].

Bit allocation is applied at source coding stage, where given total number of bits allocation to each transform coefficient in such a way that minimize the distortion in terms of MSE. The Rate Distortion(R-D) theory is a classical framework for bit allocation [2]. For arbitrary set of quantizers optimal solution of bit allocation has been defined by Shoham and Gersho [3]. This algorithm has high computational complexity. Various bit allocation model have been proposed based on information theory [4]-[7].

In JPEG image transmission system, various error control

Manuscript received October 30, 2014; revised May 10, 2015

Jigisha N. Patel is with the S V National Institute of Technology, Surat, Gujarat, India (e-mail: jnpatel@ eced.svnit.ac.in).

Suprava Patnaik was with S V National Institute of Technology, Surat. $\mathrm{He}$ is now with the Department of Electronics Engineering, Xaviers Institute of Engineering, Mahim, Mumbai, India (e-mail: ssp@eced.svnit.ac.in).

Saraiya Mansi is with the Electronics Engineering Department, S V National Institute of Technology, Surat, Gujarat, India (e-mail mansi.saraiya11@gmail.com). coding scheme like Layered coding with UEP, Error resilience, Error concealment etc. are used to reduce the error. This paper proposed layered based unequal error protection. So priority based channel protection can be allocated and that is the motivation to develop UEP. Using UEP significant gain performance improvement can be obtained compare to Equal Error Protection (EEP) [8]-[10]. The required steps for unequal error protection are: proper data partition and proper channel protection assignment according to importance of bit stream.

This paper is organised as follows: Section II describes overall system block diagram. Section III shows optimal bit allocation algorithm with quantizer design. Section IV discusses simulation results for transmission system with unequal error protection. Section $\mathrm{V}$ concludes the work.

\section{OVERVIEW OF SYSTEM}

The system block diagram is shown in Fig. 1. Input image is divided into non overlap $8 \times 8$ blocks. DCT transform is applied for each block. Blocks are classified into three classes using block classification algorithm Equal Mean Normalized Standard Deviation (EMNSD) proposed by [11]. The modified EMNSD is representing in [12]. The class 1, class 2 and class 3 of block classification assigned the name as smooth, edge and texture. Fig. 2 shows EMNSD block classification result for two classes and three classes for Barbara image. DC Coefficients are separated from each block and $\mathrm{AC}$ coefficients are classified as smooth $\mathrm{AC}$ coefficient $\left(\mathrm{AC}_{\mathrm{S}}\right)$, edge $\mathrm{AC}$ coefficient $\left(\mathrm{AC}_{\mathrm{E}}\right)$ and texture $\mathrm{AC}$ coefficient $\left(\mathrm{AC}_{\mathrm{T}}\right)$.

Optimum bit allocation and according to quantizer is designed for these three class of blocks. The quantized data follows standard procedure like zigzag, Run Length and Huffman coding. Source Coded data passes through Rate Compatible Puncture Convolutional Coder (RCPC) [10] which is suitable for UEP application. In JSCC [13] system total transmit rate will be fixed. The goal of JSCC system is distribute total transmission rate between source rate and channel rate such a way that end to end distortion is minimized. The source code rate further distributed among three classes using bit allocation algorithm. The compressed bit stream of each class has assigned the channel protection according to its significance in decoding the image. Among the entire bit stream the DC coefficient bitstream has applied highest protection compared to AC coefficient bit stream. Among AC coefficient bitstream texture block AC bitstream is highly protected. The system is simulated for fixed transmission rate $\left(\mathrm{R}_{\text {Total }}\right)$ and channel condition. 


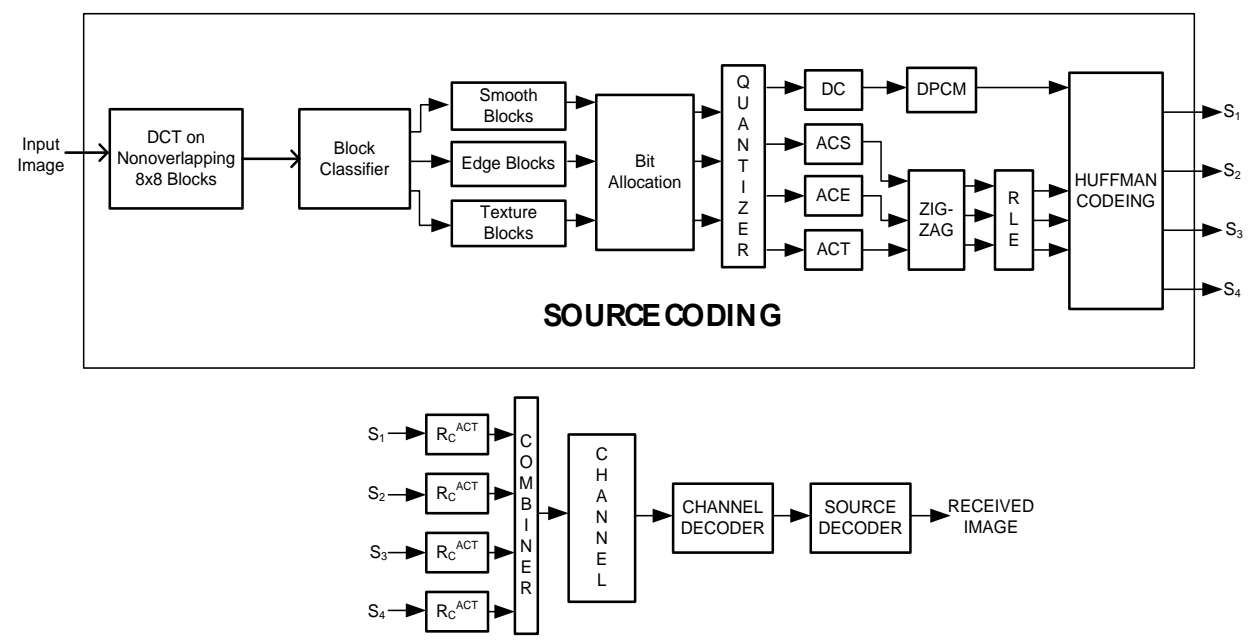

Fig. 1. Image transmission system block diagram.

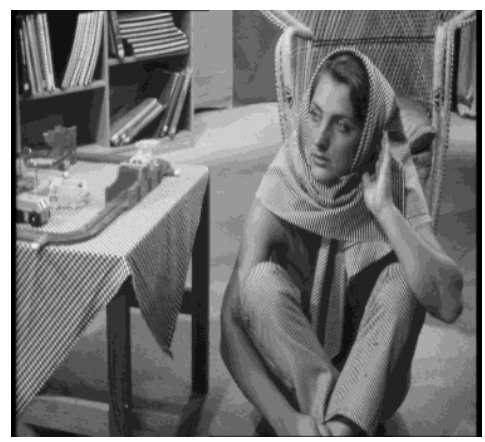

(a)

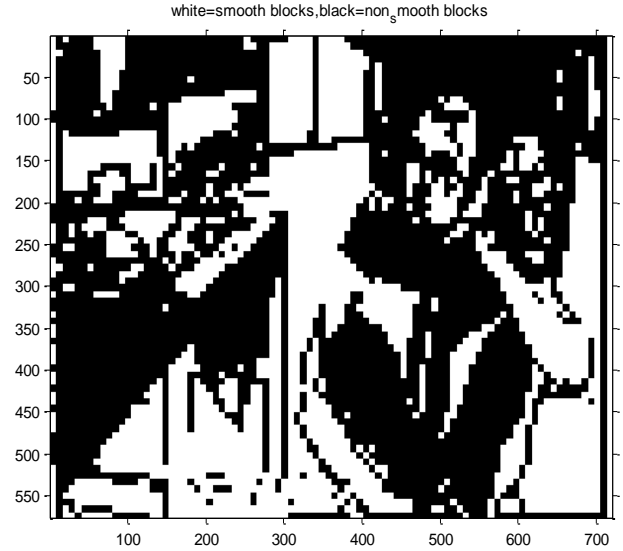

(b)

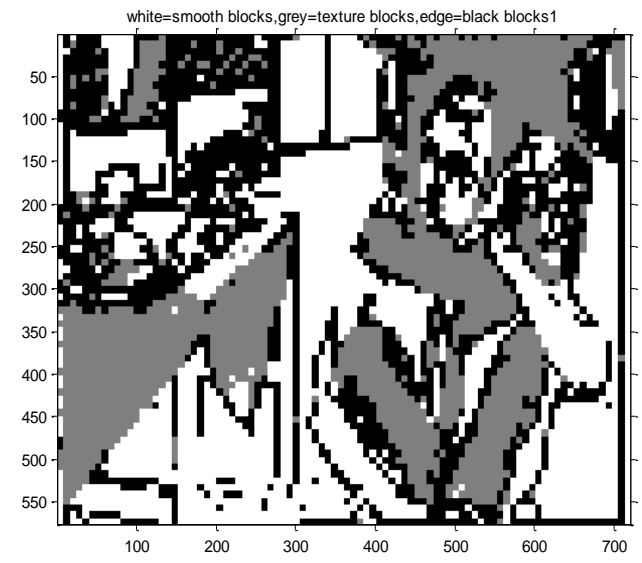

(c)

Fig. 2. (a) Original image Barbara, (b) Two classes blocks classification, (c) Three classes block classification.

The session III highlights the optimum bit allocation algorithm.

\section{Bit AllocAtion Algorithm}

Once the blocks are classified the next task is to allocate optimum bit according to block activity. Image adaptive coding is suggested in [14]. Actually the bit allocation required at two different stages

1) The average source bit rate is allocated among the $K$ classes and

2) Bit allocation of Kth class rate to each transform coefficient

The average source bit rate is allocated among the $K$ classes such that [7]

$$
\sum_{k=1}^{3} w_{k} R_{k}=R_{s} \quad \sum_{k=1}^{3} w_{k}=1
$$

here $w_{k}$ is probability of particular class, $R_{S}$ is total source rate and $R_{k}$ is rate allocated to $k^{\text {th }}$ class. The $R_{k}$ can be found as

$$
R_{k}=R_{s}+\frac{1}{2} \log _{2} \frac{A_{k}}{\left[\prod_{i=0}^{k-1} A_{k}^{w_{i}}\right]}
$$

If $M=8 \times 8$ block size and $\sigma_{k, j}^{2}$ is the variance, than $A_{k}$ can be calculate as

$$
A_{k}=\varepsilon^{2}\left[\prod_{j=0}^{M-1} \sigma_{k, j}^{2}\right]^{\frac{1}{M}}
$$

Once the rate allocation at each class is allocated, next task is allocating $R_{k}$ into each coefficient of $k^{\text {th }}$ class. In lossy compression DCT transform coefficient follows the quantization. The $R_{k, i}$ is the rate of $k^{\text {th }}$ class of $i^{\text {th }}$ transform coefficient. Using Langrage multiplication [7] method it can be calculated as

$$
R_{k, i}=R_{k}+\frac{1}{2} \log _{2}\left\{\frac{\sigma_{k}^{2}}{\left(\prod_{j=1}^{M} \sigma_{j}\right)^{\frac{1}{M}}}\right\}
$$

Thus, the number of bits for the $k^{\text {th }}$ quantizer is equal to the average bit rate plus a quantity that depends on the ratio of its input variance to the geometric mean of the variances of all the coefficients. In our simulation $K=3$ class and average bit 
rate $R_{s}$ first divided into $R_{k}$ as smooth, edge and texture. The simulation results for Barbara image with $R_{S}=1$ bpp is shown in Table I.

TABLE I: BIT ALLOCATION FOR (A) SMOOTH BLOCKS (B) TEXTURE BLOCKS(C) EDGE BLOCKS

\begin{tabular}{|l|l|l|l|l|l|l|l|}
\hline 13 & 3 & 2 & 1 & 0 & 0 & 0 & 0 \\
\hline 3 & 1 & 0 & 0 & 0 & 0 & 0 & 0 \\
\hline 1 & 0 & 0 & 0 & 0 & 0 & 0 & 0 \\
\hline 0 & 0 & 0 & 0 & 0 & 0 & 0 & 0 \\
\hline 0 & 0 & 0 & 0 & 0 & 0 & 0 & 0 \\
\hline 0 & 0 & 0 & 0 & 0 & 0 & 0 & 0 \\
\hline 0 & 0 & 0 & 0 & 0 & 0 & 0 & 0 \\
\hline 0 & 0 & 0 & 0 & 0 & 0 & 0 & 0 \\
\hline
\end{tabular}

\begin{tabular}{|l|l|l|l|l|l|l|l|}
\hline 13 & 10 & 8 & 6 & 6 & 4 & 4 & 3 \\
\hline 8 & 6 & 5 & 4 & 3 & 3 & 2 & 2 \\
\hline 6 & 5 & 4 & 3 & 3 & 3 & 2 & 2 \\
\hline 5 & 3 & 3 & 3 & 4 & 3 & 2 & 2 \\
\hline 4 & 3 & 2 & 2 & 3 & 3 & 2 & 2 \\
\hline 4 & 3 & 2 & 0 & 1 & 1 & 1 & 1 \\
\hline 2 & 2 & 0 & 0 & 0 & 0 & 0 & 0 \\
\hline 0 & 0 & 0 & 0 & 0 & 0 & 0 & 0 \\
\hline
\end{tabular}

\begin{tabular}{|l|l|l|l|l|l|l|l|}
\hline 11 & 5 & 4 & 5 & 5 & 3 & 2 & 1 \\
\hline 5 & 4 & 5 & 3 & 3 & 2 & 2 & 1 \\
\hline 4 & 4 & 4 & 2 & 3 & 3 & 2 & 2 \\
\hline 5 & 3 & 3 & 2 & 5 & 5 & 2 & 1 \\
\hline 4 & 2 & 2 & 2 & 4 & 3 & 2 & 2 \\
\hline 3 & 5 & 1 & 1 & 1 & 0 & 2 & 2 \\
\hline 3 & 1 & 1 & 1 & 0 & 0 & 0 & 0 \\
\hline 0 & 0 & 0 & 0 & 0 & 0 & 0 & 0 \\
\hline
\end{tabular}

The next step is to design the quantizer based on individual bits obtained for each transform coefficient from optimal bit allocation. The quantizer design can be uniform or non-uniform. The optimum quantizer design Lloyd Max Quantizer is used in this simulation. The Fig. 3 shows the performance by bit rate versus quantizer parameter SNR. Fig. 4 compares the performance with uniform quantizer.

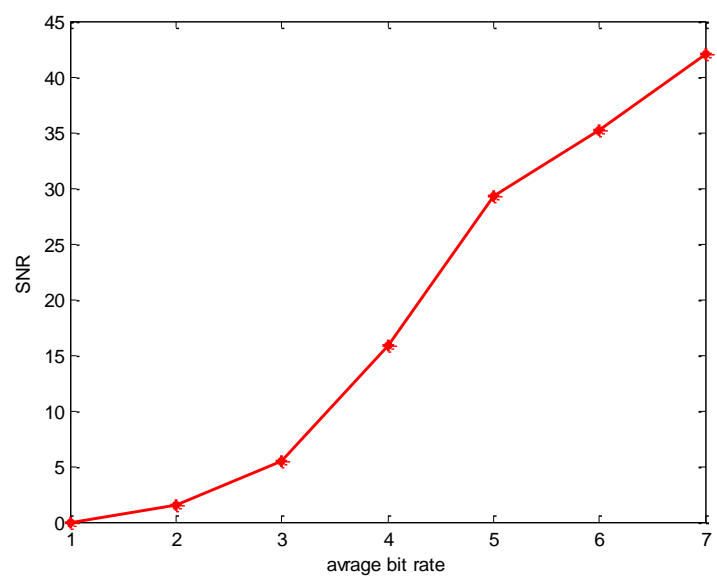

Fig. 3. Lloyd's quantizer performance.

\section{UNEQUAL ERROR PROTECTION AND SimULATION RESULTS}

In 2001 Wei Xiang and Steven [9] has proposed Unequal Error Protection (UEP) method to JPEG image transmission using Turbo codes. The significance of smooth, edge and texture AC coefficient bit stream in received image can be decide protection level. Consider error in smooth region as
$\mathrm{Pe}_{\text {ACS }}$ error in edge region as $\mathrm{Pe} \_\mathrm{ACE}$, error in texture region as Pe_ACт. Find the effect of smooth block bitstream in decoded image by setting $\mathrm{Pe}_{-\mathrm{ACT}}=\mathrm{Pe} \_\mathrm{ACE}=0$. Vary the error $\mathrm{Pe} \_\mathrm{ACS}$ and calculate amount of distortion. Repeat for edge blocks and texture blocks also. The following Fig. 5 shows for channel sensitivity curve for Barbara image.

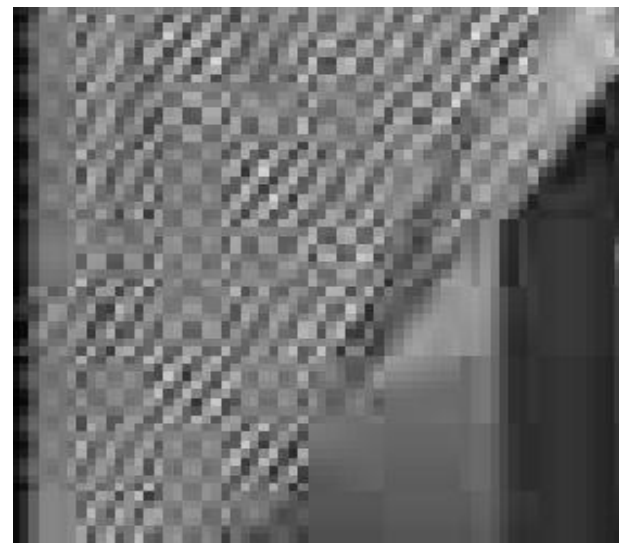

(a)

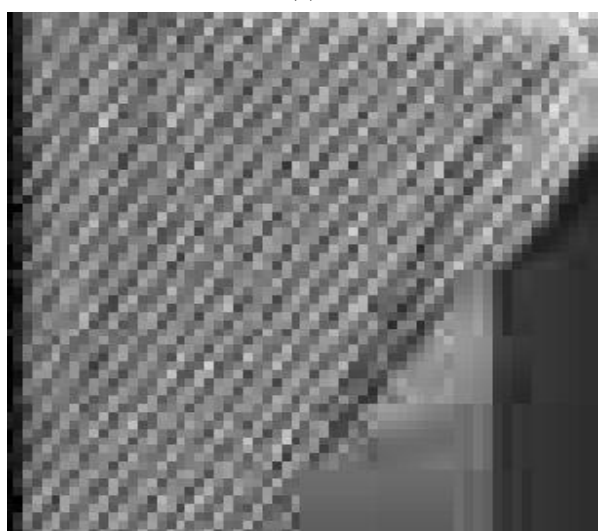

(b)

Fig. 4. Zoom region of Barbara image for (a) uniform quantizer (b) Llyod max quantizer.

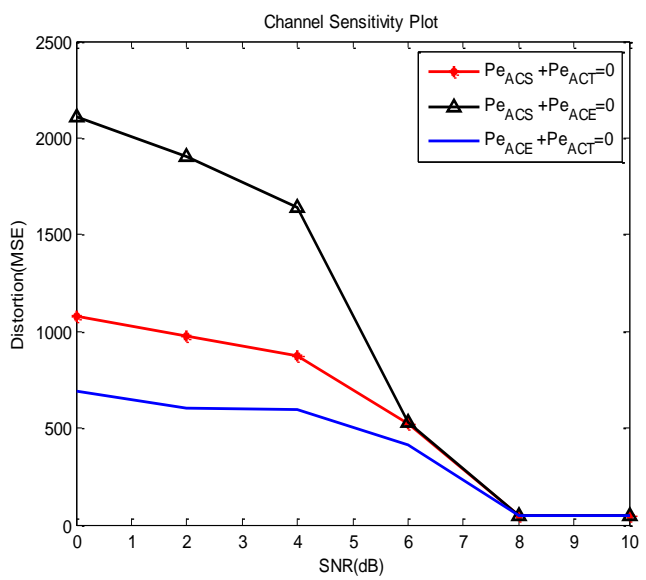

Fig. 5. Channel sensitivity curve for Barbara image.

When the same protection is assigned to all encoded source layer bits regardless their significance, the method is called Equal Error Protection (EEP). The method of modulating the amount of channel coding based on the required level of protection is known as Unequal Error Protection (UEP) [5], [9]. The simulation results shown in Table II for channel condition $\mathrm{SNR}=2 \mathrm{~dB}$ of $\mathrm{AWGN}$ channel. Here $\mathrm{PSNR}_{\mathrm{BA}}$ stands for system with optimum bit allocation and PSNR stands for without bit allocation. 
The UEP symbol is used for conventional unequal error protection method and $\mathrm{UEP}_{\mathrm{SET}}$ for proposed classified method. The symbol $R_{C}{ }^{D C}, R_{C}{ }^{A}, R_{C}{ }^{S}, R_{C}{ }^{E}, R_{C}{ }^{T}$ indicates channel code rate for DC, AC coefficients, smooth block bitstream, edge block bitstream and texture block bitstream. It is observed using proper bit allocation received image quality can be improved. Following Fig. 6 shows the comparison of unequal error protection with and without bit allocation for different $R_{\text {Total }}$. It is observed that for lower SNR value performance improves compare to without bit allocation.

\begin{tabular}{|c|c|c|c|c|c|c|c|c|c|}
\hline Image & Method & $R_{S}$ & \multicolumn{4}{|c|}{$R_{c}$} & $R_{\text {Total }}$ & $P S N R(d B)$ & $\operatorname{PSNR}_{B A}(d B)$ \\
\hline \multirow[t]{5}{*}{ Barbara } & EEP & 0.76 & \multicolumn{4}{|c|}{$8 / 11$} & 1.2 & 7.63 & 11.45 \\
\hline & \multirow[t]{2}{*}{ UEP } & \multirow[t]{2}{*}{0.80} & \multicolumn{2}{|c|}{$R_{c}{ }^{D C}$} & \multicolumn{2}{|c|}{$R_{c}{ }^{A C}$} & \multirow[t]{2}{*}{1.2} & \multirow[t]{2}{*}{15.07} & \multirow[t]{2}{*}{13.96} \\
\hline & & & \multicolumn{2}{|c|}{$8 / 15$} & \multicolumn{2}{|c|}{$8 / 10$} & & & \\
\hline & \multirow[t]{2}{*}{$U E P_{S E T}$} & \multirow[t]{2}{*}{0.69} & $R_{c}{ }^{D C}$ & $R_{c}{ }^{S}$ & $R_{c}{ }^{E}$ & $R_{c}^{T}$ & \multirow[t]{2}{*}{1.21} & \multirow[t]{2}{*}{20.90} & \multirow[t]{2}{*}{22.32} \\
\hline & & & $8 / 15$ & $8 / 10$ & $8 / 12$ & $8 / 11$ & & & \\
\hline Image & Method & $R_{S}$ & & & & & $R_{\text {Total }}$ & $P S N R$ & $P S N R_{B A}$ \\
\hline \multirow[t]{5}{*}{ Cameraman } & EEP & 0.8513 & \multicolumn{4}{|c|}{$8 / 10$} & 1.19 & 9.05 & 5.57 \\
\hline & \multirow[t]{2}{*}{ UEP } & \multirow[t]{2}{*}{0.8860} & \multirow{2}{*}{\multicolumn{2}{|c|}{$\begin{array}{l}R_{c}{ }^{D C} \\
8 / 15\end{array}$}} & \multicolumn{2}{|c|}{$R_{c}^{A C}$} & \multirow[t]{2}{*}{1.19} & \multirow[t]{2}{*}{13.46} & \multirow[t]{2}{*}{13.40} \\
\hline & & & & & & & & & \\
\hline & \multirow[t]{2}{*}{$U E P_{S E T}$} & \multirow[t]{2}{*}{0.8623} & $R_{c}{ }^{D C}$ & $R_{c}^{S}$ & $R_{c}^{E}$ & $R_{c}{ }^{T}$ & \multirow[t]{2}{*}{1.19} & \multirow[t]{2}{*}{14.94} & \multirow[t]{2}{*}{16.17} \\
\hline & & & $8 / 15$ & $8 / 8$ & $8 / 10$ & $8 / 9$ & & & \\
\hline Image & Method & $R_{S}$ & & & & & $R_{\text {Total }}$ & $P S N R$ & $P S N R_{B A}$ \\
\hline \multirow[t]{5}{*}{ Baboon } & EEP & 0.8951 & \multicolumn{4}{|c|}{$8 / 11$} & 1.4 & 11.6 & 9.71 \\
\hline & \multirow[t]{2}{*}{ UEP } & \multirow[t]{2}{*}{0.9541} & & & & & 1.4 & 15.89 & 13.67 \\
\hline & & & & & & & & & \\
\hline & $U E P_{S E T}$ & 0.839 & $R_{c}^{D C}$ & $R_{c}^{S}$ & $R_{c}{ }^{E}$ & $R_{c}{ }^{T}$ & 1.4 & 19.87 & 20.30 \\
\hline & & & $8 / 15$ & $8 / 10$ & $8 / 12$ & $8 / 11$ & & & \\
\hline
\end{tabular}

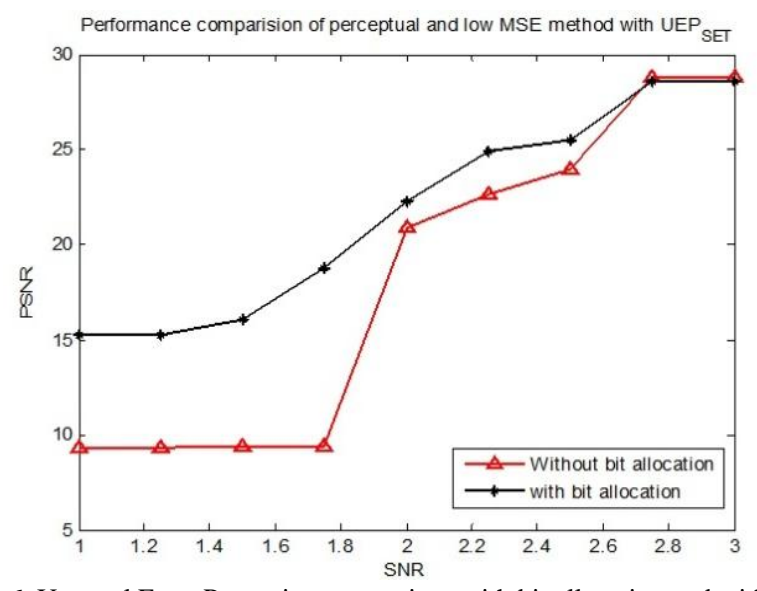

Fig. 6. Unequal Error Protection comparison with bit allocation and without bit allocation for different SNR.

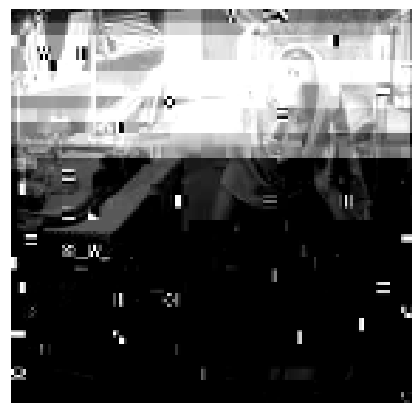

(a)

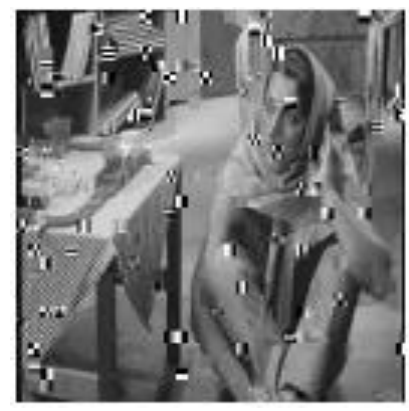

(c)

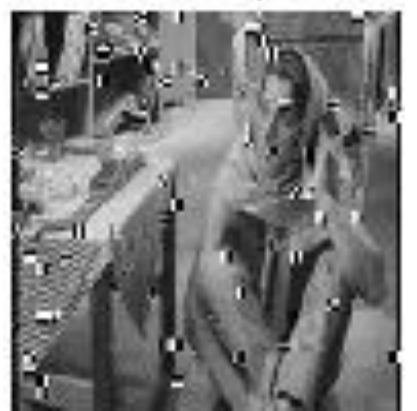

(b)

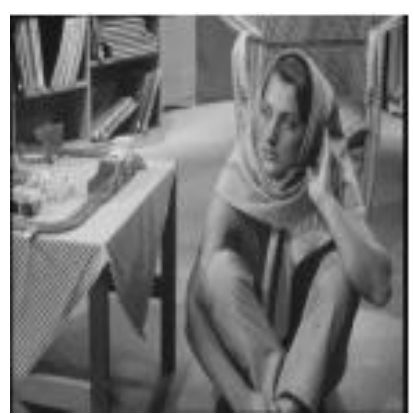

(d)

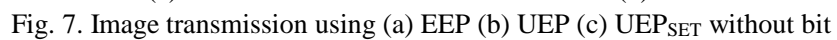

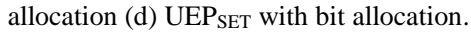

The following Fig. 7 shows the comparison of visual quality of Barbara image with different error protection condition.

\section{CONCLUSION}

Bit allocation is problem for source coding. The overall quality can be improved using proper classification and bit allocation method. The transmission of compressed image is very sensitive to channel noise. So received quality can be improved using one of error control coding scheme like unequal error protection. For fair comparison all simulation results for EEP, UEP and $\mathrm{UEP}_{\mathrm{SET}}$ are compared for fixed transmission rate and channel condition.

The simulation results shows that using this algorithm approximately 3-4 dB improvement compare to conventional UEP.

\section{REFERENCES}

[1] G. K. Wallace, "The JPEG still picture compression standard," ACM Communication, vol. 34, no. 4, pp. 31-45, 1991.

[2] A. Ortega and Ramchandran, "Rate distortion methods for image and video compression," IEEE Signal Processing Magazine, vol. 15, no. 6, pp. 23-50, 1998.

[3] Y. Shoham and A. Gersho, "Efficient bit allocation for an arbitrary set of quantizers," IEEE Transaction Acoust, Speech, Signal Processing, pp. 1445-1453, Sept.1988.

[4] D. Akopian, "On optimal bit allocation for classification: Based source dependent transform coding," Research Letter in Signal Processing, Hindawi Publishing Corporation, pp. 1-5, 2008.

[5] P. H. Westerink, J. Biemond, and D. E. Boekee, "An optimal bit allocation algorithm for band coding," in Proc. the IEEE International conference on Acoustics, Speech and Processing, USA, vol. 2, April 1988.

[6] V. V. Gohokar and V. N. gohokar, "Fuzzy based optimum bit allocation for Rate control in video Compression," IETE Journal of Research, vol. 56, issue 4, pp. 213-218, Jul.-Aug. 2010.

[7] H. Watanabe and S. Singhal, "Bit allocation and rate control based on Human visual sensitivity for Intraframe Coders," in Proc. IEEE International Conference on Speech, and Signal Processing, 1992.

[8] T. P. Fodder and K. M. S. Soyjaaudah, "Robust JPEG image transmission using unequal error protection and code combining," 
International Journal of Communication Systems, vol. 21, pp. 1-24, 2007.

[9] W. X. Barbulescu and S. Pietrobon, "Unequal error protection applied to JPEG image transmission using turbo Codes," IEEE Trans. on Communication, vol. 46, Dec. 1998.

[10] J. Hagenauer, "Rate compatible puntured convolutional codes and their application," IEEE Trans. on Communication, vol. 36, pp. 389-400, April 1988.

[11] R. L. Joshi, H. Jafarkhani, J. H. Kasner, T. R. fisher, N. Farvadin, M. W marcclin, and R. H. Bamberger, "Comparison of diffrent methods of classification in subband coding of images," IEEE Trans. on Image Processing, vol. 6, pp. 1473-1485, Nov. 1997.

[12] J.-F. Cai and C. W. Chen, "Uniform threshold TCQ with block classification for image transmission over noisy channels," IEEE Trans. on Circuits and Systems for Video Technology, vol. 11, no. 1, January 2001.

[13] S. M. F. sheikh, H. R. Heath et al., "A joint source channel distortion model for JPEG compressed image," in Proc. the 2004 International Conference on Image Processing, Singapore, Oct. 24-27, 2004, pp. 3249-3252.

[14] W. H. Chen and C. H. Smith, "Adaptive coding and monochrome and colour images," IEEE Transaction Communication, vol. 25, pp. 1285-1292, Nov. 1977.

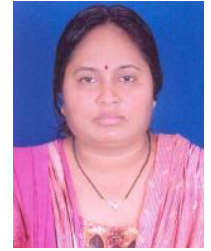

Jigisjha N. Patel received the M.Tech degree in communication systems from the Electronics Engineering Department, Sardar Vallabhbhai National Institute of Technology, Surat in 2007. Currently she is pursuing her Ph.D. degree from SVNIT in the area of image processing and communication. Her research area includes image/video compression, wireless communication. She is an assistant professor in the Electronics Department, SVNIT Surat.

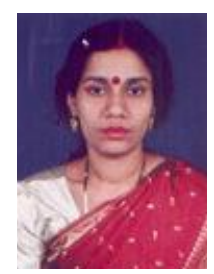

Suprava Patnaik has completed her Ph.D. degree from IIT, Kharagpur. Her main research areas are signal processing, image processing and computer vision. She was a professor and the head at SVNIT, Surat and currently working as a professor the head in Xavier Institute of Engineering, Mumbai. She has published more than 50 research papers in national as well as international journals and conference proceedings. She is a member of IEEE, and life member of other professional societies. She has delivered various expert lecture in international conferences, workshops etc.

Mansi Sariya has completed her M.Tech. degree from SVNIT, Surat in 2013. Her research interests are image processing and wireless communication. 